\title{
ATENDIMENTO FONOAUDIOLÓGICO EM GRUPO
}

\author{
Speech therapy group care
}

\author{
Manuela Luchesi Brazil Araújo (1), Regina Maria Ayres de Camargo Freire (2)
}

\begin{abstract}
RESUMO
Tema: atendimento fonoaudiológico em grupo na área da Fonoaudiologia. Para sua realização, visitou-se os textos publicados em revistas científicas da área, nos últimos 15 anos, e os capítulos de livros e dissertações que se detiveram em tematizar o atendimento fonoaudiológico em grupo. Objetivo: identificar, entender e discutir: 1) os princípios teóricos que justificam a indicação do atendimento em grupo, que o estruturam e o sustentam de maneira a explicitar como a dinâmica do grupo age sobre os sintomas ; 2) os critérios para a composição e formação dos grupos e, principalmente, 3 ) a intervenção do fonoaudiólogo e a possibilidade de eficácia de sua terapêutica. Conclusão: o levantamento bibliográfico identificou diferentes práticas fonoaudiológicas em grupo, atreladas a diversas perspectivas teóricas. No que diz respeito aos critérios de formação, à função do fonoaudiólogo no grupo, à prevalência do atendimento em grupo em relação ao individual, houve divergências de opiniões, dado que os textos visitados, não (cor)responderam com a consistência teórica esperada. Tal resultado se deve ao fato de haver uma certa adoção irrefletida de conceitos de outras áreas, sem argumentação e sem a reestruturação que sua transposição para a clínica fonoaudiológica, exigiria. Sugere-se que estas incoerências ocorrem porque não se evidencia a preocupação em articular os estudos teóricos aos elementos que configuram a clínica fonoaudiológica, a saber: a semiologia, a etiologia, a diagnóstica e a terapêutica.
\end{abstract}

DESCRITORES: Fonoaudiologia; Transtornos da Linguagem; Terapia da Linguagem; Grupo Paritário

\section{INTRODUÇÃO}

$\mathrm{O}$ atendimento Fonoaudiológico em grupo tem início com o objetivo de suprir a grande demanda para atendimento individual ${ }^{1}$. Uma busca à literatura fonoaudiológica aponta que as propostas de grupos terapêuticos tiveram início no final dos anos 80, motivadas, grandemente, pela inserção do fonoaudiólogo na Saúde Pública. Já sua investigação vem sendo tratada desde a década de 90.

Da inauguração como forma de atender a muitos em menos tempo, dada a relação desigual entre demanda da população e oferta de mão de obra fonoaudiológica, até a prática comum nos dias de hoje, o atendimento em grupo sofreu grandes

(1) Fonoaudióloga; Mestre em Fonoaudiologia pela Pontifícia Universidade Católica de São Paulo - PUCSP.

(2) Fonoaudióloga; Professora titular do Departamento de Clínica Fonoaudiológica da Pontifícia Universidade Católica de São Paulo - PUCSP; Pós doutora pela Universidade de São Paulo - PUCSP.

Conflito de interesses: inexistente mudanças. Estas foram devidas principalmente ao novo cenário da Saúde Pública com a entrada do Programa de Saúde da Família, o que desencadeou a contratação de fonoaudiólogos para as UBS (Unidade Básica de Saúde) e para os NASF (Núcleo de Apoio de Saúde).

Ressalte-se que o Atendimento Fonoaudiológico em Grupo a que nos referimos aqui, relacionase ao que é chamado, dentre outros nomes, de Grupo Terapêutico Fonoaudiológico ou de Terapêutica Fonoaudiológica Grupal, o que é diferente de Grupo Educativo, Grupo de Orientação (Familiar ou Escolar), Grupo de Discussão, Grupo de Reflexão, ou Grupo de Apoio, estes vinculados ao ensino e aprendizagem e à prevenção e promoção de saúde, modos de atendimento que não serão tematizados por fugirem ao escopo deste trabalho.

O objetivo deste trabalho é identificar, entender e discutir: 1) os princípios teóricos que justificam a indicação do atendimento em grupo, que o estruturam e o sustentam de maneira a explicitar como a dinâmica do grupo age sobre os sintomas; 2) os critérios para a composição e formação dos grupos 
e, principalmente, 3) a intervenção do fonoaudiólogo e a possibilidade de eficácia de sua terapêutica.

\section{MÉTODO}

Para sua realização buscou-se os textos publicados, nos últimos 15 anos, sob a forma de artigos, capítulos de livros e dissertações que se detiveram em tematizar o atendimento fonoaudiológico em grupo. Os artigos foram extraídos de Revistas específicas da área Fonoaudiológica, a saber: Sociedade Brasileira de Fonoaudiologia, Pró-Fono, Distúrbios da Comunicação e Fonoaudiologia Brasil. As bases de dados utilizadas foram LILACS, Medline e Scielo. Foram excluídos os artigos, livros e textos que não se dedicaram ao tema destacado acima.

\section{REVISÃO DA LITERATURA}

Os trabalhos visitados consideram os atendimentos em grupo como experiências positivas e a maior parte sustenta-se na perspectiva sóciohistórico-cultural que toma por base os estudos de Vygotsky e deriva de alguns princípios defendidos por Bion, Pichon-Riviére, Kaës, entre outros, sobre essa forma de atendimento.

Os trabalhos encontrados foram organizados em categorias para responder às três questões colocadas acima, as quais foram formuladas a partir da introdução, com a finalidade de produzir uma teorização sobre o processo clínico advinda da análise dos efeitos terapêuticos do atendimento em grupo sobre os sintomas de fala dos participantes do próprio grupo.

$\mathrm{Na}$ primeira categoria, denominada pressupostos teóricos do atendimento fonoaudiológico em grupo, serão apresentados e discutidos alguns trabalhos científicos realizados no campo fonoaudiológico com a finalidade de traçar o percurso teórico que lhes serve de base, articulando-o à dinâmica do atendimento fonoaudiológico em grupo.

O estudo de Freitas, Lacerda e Panhoca ${ }^{2}$ marca o início da teorização sobre o "atendimento fonoaudiológico em grupo" tendo como suporte a corrente sócio-histórico-cultural defendida por Vygotsky. As autoras afirmam que $\mathrm{o}$ atendimento fonoaudiológico em grupo é um contexto social em que os sujeitos, com seu legado cultural, irão amadurecer, desenvolver-se e partilhar os conhecimentos historicamente construídos, além de desenvolver o outro, e, assim, a cultura.

As autoras adotam alguns princípios defendidos por Bion e Pichon-Rivière, sobre o atendimento em grupo. De Bion extraem os princípios de constituição do grupo, de Pichon-Rivière que o grupo é um conjunto de pessoas reunidas em torno de uma tarefa comum e o resultado da dialética entre os movimentos de horizontalidade (história do grupo) e verticalidade (histórias pessoais). Entendem o grupo como um meio facilitador numa "atividade onde a linguagem pode ser trabalhada em sua plenitude (...) é levada a desenvolver-se muito mais rápida e reflexivamente do que no atendimento terapêutico individual" (p.59), objetivo que, de um lado, escapa aos autores tomados como base e, de outro, não é aprofundado.

O grupo é considerado o contexto sociocultural "adequado" para o desenvolvimento lingüístico, "poderoso gerador e propulsor de atividades lingüísticas e sociais" (p.64), no qual a "linguagem pode ser trabalhada fácil e rápida" (p. 57). Para que ocorra desenvolvimento lingüístico rápido e eficiente, é preciso que seja criada a necessidade do uso da linguagem, o que é, por si só, inerente ao funcionamento do grupo enquanto contexto terapêutico e atividade social.

Panhoca ${ }^{3}$, Panhoca e Leite ${ }^{4}$, Penteado et $a^{5}$, Panhoca e Penteado ${ }^{6}$, Mourão et al ${ }^{7}$, Machado, Berberian e Massi ${ }^{8}$ seguem os mesmos pressupostos teóricos dos estudos de Freitas, Lacerda e Panhoca ${ }^{2}$, com a introdução dos conceitos: organizadores culturais, subjetividade, vínculo e papéis, que serão explicitados a seguir.

Além disso, e ainda nesta abordagem, Panhoca ${ }^{3}$ faz uso do conceito de "organizadores culturais" de Luria e Yudovich - fenômenos que se iniciam inter-psíquicos e depois tornam-se intra-psíquicos - e utiliza a linguagem como instrumento ao considerar o grupo terapêutico como o lugar em que as experiências lingüístico-comunicativas são favorecidas e ocorrem inicialmente entre pessoas (intermentais) para tornarem-se pessoais (intramental). Nesse contexto, considera-se o estabelecimento e o cumprimento de regras e de rotinas para propiciar um ambiente adequado que beneficie os integrantes do grupo.

Panhoca e Leite 4 inauguram as primeiras questões relacionadas à intersubjetividade ao apontar que terapeuta e paciente estão em posição dialógica na qual dizeres se cruzam, se afetam e produzem outros sentidos. Citam Bion e referem-se ao grupo como "o lugar de construções criativas no campo das relações sociais" (p.293).

Panhoca e Penteado ${ }^{6}$ fazem referência aos estudos de Pichon e Zimerman sobre a idéia de vínculo, justificando-o como essencial para compreender o sujeito no grupo.

Para Machado, Berberian e Massi ${ }^{8}$, a abordagem terapêutica grupal sustenta-se na singularidade do sujeito, com base na linguagem discursiva da perspectiva interacionista e na proposta da 
corrente sócio-histórica e, ainda, nos estudos realizados por Zimerman, Pichon-Rivière e Yozo para refletir sobre as relações que se dão no interior do grupo. De Zimerman extraem a distinção entre grupo e agrupamento e destacam que o grupo terapêutico possibilita a criação de vínculos intersubjetivos onde afloram os fenômenos de identificação, ressonância, pertencimento e continência. De Pichon-Rivière alçam os conceitos de vínculo e de papéis. Utilizam a idéia de vínculos intersubjetivos, proposta por Käes, porque concordam que as relações entre os sujeitos produzem formações e processos psíquicos específicos que se organizam a partir daquilo que cada sujeito projeta no grupo.

Desta forma, as autoras concluem que a transformação do agrupamento em grupo se dá pelo vínculo e pelos papéis que os sujeitos assumem na dinâmica do processo grupal e, para a configuração do grupo, é fundamental a promoção de discussões sobre o porquê de estar em grupo e, portanto, em torno de aspectos relacionados aos limites, sofrimentos e problemas vividos pelos sujeitos.

Em uma outra abordagem, Hugenneyer e Oliveira ${ }^{9}$ baseiam-se em uma visão desenvolvimentista de linguagem e tomam o grupo como propiciador desta. Afirmam que neste atendimento os pacientes têm a possibilidade de perceber a sua linguagem, suas atitudes e a si mesmo pelo movimento de uma outra pessoa, provocando um deslocamento determinante no processo terapêutico. As autoras concluem que a dinâmica grupal deriva de um grupo que se configura com suas particularidades e sem interferência, a priori, da terapeuta e apenas afirmam a terapêutica como uma experiência positiva.

Diante de outros pressupostos, Friedman e Passos ${ }^{10}$ destacam o caráter imprescindível dos grupos como dispositivos de mobilização das dinâmicas inter e intra-subjetivas, as quais são importantes à superação de problemas. Nesta direção, as autoras entendem que o grupo ressalta o papel do outro e facilita a expressão de alterações de linguagem.

Afirmam que o vínculo tem um efeito na constituição da subjetividade e na linguagem dos pacientes. Esta constituição se dá a partir da vivência do sujeito no grupo onde compartilha a sua subjetividade com o outro. Dessa forma, consideram que o sujeito tem uma dimensão grupal intra e inter psíquica, como defende Kaës.

Já Passos ${ }^{11}$ toma as concepções de sujeito e linguagem como fundantes do método clínico fonoaudiológico. Neste trabalho, a autora busca inspiração na psicologia, em particular nos princípios técnicos de Zimerman, para estruturar os princípios que regem o funcionamento do grupo na
Fonoaudiologia como: comunicação (verbal e não verbal), atividade intersubjetiva, atividade interpretativa, desempenho de papéis e vínculos.

A autora defende que o grupo e seu funcionamento são construídos na prática e que as características do atendimento fonoaudiológico em grupo são adquiridas no decorrer do funcionamento. Diante disso, a linguagem é o recurso técnico da intervenção fonoaudiológica e no que diz respeito a esse tipo de intervenção, o fonoaudiólogo utiliza-se da atividade interpretativa da fala dos sujeitos para que ocorram os deslocamentos de posições discursivas. Além disso, quando um componente do grupo tem sua fala interpretada não deixa de interpretar outras falas, movimento que leva à reestruturação de sua fala, e que acarretará deslocamentos e deslizamentos discursivos no interior do grupo.

As relações intersubjetivas podem trazer à tona o movimento lingüístico-discursivo dos pacientes, uma vez que nos grupos "várias são as bocas e vários são os ouvidos, o que cria diversas redes e diversas formas de interpretação", diferentemente do atendimento individual no qual a "relação dialógica se dá entre terapeuta e paciente" (p.65).

A análise crítica da categoria pressupostos teóricos do atendimento fonoaudiológico em grupo, é finalizada aderindo-se à afirmação de Corrêa ${ }^{1}$ no que concerne à falta de "problematização da definição de grupo" (p.47) e de reflexão sobre os diversos paradigmas teóricos que subjazem este tipo de atendimento fonoaudiológico, visto que os trabalhos visitados indicam os pressupostos acessados, porém, não sustentam ou estruturam a sua prática grupal em articulação com esses pressupostos.

A segunda categoria, que se denominou critérios internos ao grupo, destaca os trabalhos que discorrem sobre a formação e a composição de um grupo terapêutico.

Para iniciar a análise dessa categoria, serão apresentados alguns trabalhos que tem como característica para a constituição e a formação de grupo, os critérios de homogeneidade, a saber, a faixa etária e/ou patologia semelhante, e aqueles que, em contrapartida, assumem critérios de heterogeneidade, ou seja, patologias e faixas etárias diferentes.

Entre os trabalhos que defendem a homogeneidade está 0 de Hugenneyer e Oliveira ${ }^{9}$ que concordam com a crítica de Corrêa ${ }^{1}$ quando esta refere que no atendimento em grupo os participantes são manejados como nos atendimentos individuais. Isto é depreendido da forma de atuação que se dá sobre a patologia do paciente isoladamente e juntamente com os demais, além de alternar a atenção para a dificuldade de cada um 
durante as sessões. Em contraposição à Corrêa ${ }^{1}$, estas autoras afirmam que a utilização da mesma bateria de exercícios fonoaudiológicos serviria para facilitar a aprendizagem de todos os participantes do grupo e por conta disto "o critério de seleção para a formação deste grupo foi o de seus integrantes apresentarem faixa etária e hipóteses diagnósticas semelhantes" (Op. cit, 2000, p. 20).

Destacando a patologia como critério primordial, há os trabalhos de Mourão et $\mathrm{al}^{7} \mathrm{e} o$ de Freitas e Dainês ${ }^{12}$ que tratam de, respectivamente, um grupo formado por laringectomizados e um grupo formado por pacientes acometidos de Deficiência Mental e Síndrome de Down. Estes estudos não se atêm à problematização do critério utilizado para formar o grupo, ou seja, a patologia.

O trabalho de Silva et al ${ }^{13}$ justifica que "o reconhecimento da patologia no outro provoca uma modificação de comportamento, um alívio emocional e um compartilhar dos processos de atuação favorável ao bom diagnóstico" (Op. cit., 2000 , p.25). Estas autoras referem que, em sua experiência clínica com grupos, estes são organizados por deficiência, por incapacidade e por desvantagem, tendo como critério de seleção dos pacientes a patologia, a idade, o prognóstico de fala e/ou motor. Trata-se de um estudo com afirmações ou sugestões de organização de trabalho sem a apresentação de alicerces teóricos que levem em conta o objeto da Fonoaudiologia: o sujeito falante. Além disso, grupos assentados sobre o déficit deficiência, incapacidade e desvantagem - não privilegiam o sujeito e suas possibilidades.

Em outro estudo, Vilela e Ferreira ${ }^{14}$ entrevistam fonoaudiólogos que realizam terapia em grupo com portadores de distúrbios vocais e questionam as razões que os levaram a escolher esta forma de atendimento. A maioria dos fonoaudiólogos relatou que sua opção foi determinada pela grande demanda no setor público. Os critérios usados para a composição dos grupos compreendem os aspectos referentes ao distúrbio, idade, grau de alteração e, às vezes, o sexo. Os pacientes que apresentam alguma dificuldade de relacionamento com outras pessoas, paralisia vocal e os pós-cirúrgicos são encaminhados para atendimento individual.

$\mathrm{O}$ artigo de Freitas, Lacerda e Panhoca ${ }^{2}$ é um dos primeiros a utilizar o conceito de heterogeneidade, o que mostra uma oposição em relação aos critérios anteriores de homogeneidade e um avanço nas práticas em grupo. Neste artigo, estas autoras referem utilizar a avaliação fonoaudiológica como forma de escolha para a seleção dos sujeitos que irão compor o grupo. Observa-se que a heterogeneidade privilegiada na formação do grupo recai sobre os diferentes tipos de patologias de linguagem, a saber: gagueira, distúrbio articulatório, leitura e escrita e diferentes faixas etárias: idades entre 7 a 11 anos.

Em Panhoca ${ }^{3}$ assume-se que o critério básico para a seleção e para o agrupamento de elementos de um grupo é a heterogeneidade entendida como as "características socioculturais e dificuldades lingüísticas diversas" (Panhoca, 1999, p.33), justificando que as diferenças entre eles seriam a contribuição de cada um ao grupo.

Freitas, Lacerda e Panhoca 2, Panhoca 3, Panhoca e Penteado ${ }^{6}$, Penteado et $a{ }^{5}{ }^{5}$ e Machado, Berberian e Massi ${ }^{8}$ tem como critério básico para a seleção e o agrupamento de elementos de um grupo, a heterogeneidade isto é, para ingressar no grupo os sujeitos deveriam ter patologias e/ou faixas etárias diversificadas.

Panhoca e Penteado ${ }^{6}$ justificam a heterogeneidade na suposição de que esta seria complementar entre os sujeitos ao afirmar que:

"a relação social dinâmica possibilita o confronto das diferenças e conduz para a formação de identidades pessoais e grupais, já que é na relação com outras pessoas, relação mediada fundamentalmente pela linguagem que o sujeito torna-se capaz (...) de constituir sua subjetividade" (PANHOCA e PENTEADO, 6, p. 262).

Desta maneira observa-se o afastamento do foco da patologia de linguagem e seus sintomas, e a aproximação do sujeito $\mathrm{e}(\mathrm{m})$ sua dimensão sócio-histórica-cultural.

O estudo de Machado, Berberian e Massi ${ }^{8}$ refere que:

"pela heterogeneidade e diversidade socioeconômica-cultural, há possibilidades de contribuição, partilha, de complementariedade em relações aos demais membros do grupo, uma vez que a dificuldade de um pode ter sua ressignificação na/pela habilidade do outro, e o que falta a um sujeito pode estar em outro" (MACHADO, BERBERIAN e MASSI, ${ }^{8}$, p.65).

Desta forma há uma interação mútua entre o sujeito e o grupo o que amplia suas possibilidades em reviver experiências anteriores que são compartilhadas, potencializadas e internalizadas na história de cada um, "atribuindo-Ihes novas interpretações e significações" (p. 66).

A heterogeneidade permitiu novas discussões sobre a formação dos grupos, porém restritas ao deslocamento entre os participantes. Isto porque as autoras estão atreladas à abordagem 
Sociointeracionista proposta por Vygotsky, a qual reduz a linguagem a um comportamento lingüístico baseado no aporte cognitivo (relacionado à aprendizagem) e, pautadas em um modelo de clínica médica preocupam-se com falhas ambientais.

Note-se que alguns dos trabalhos que propõem critérios para a formação dos grupos de atendimento fazem parte de uma clínica fonoaudiológica submetida aos conceitos da clínica médica clássica. A clínica sobre a qual este trabalho irá se sustentar advoga a não existência de critérios de formação de grupos por estar pautada em uma concepção de linguagem que lida com o funcionamento da linguagem e com o falante, sendo este, como tal, sempre singular.

Aproximando-se de uma outra abordagem para subsidiar a clínica fonoaudiológica, o estudo de Passos ${ }^{11}$ toma o sujeito e a linguagem como esteios do método clínico fonoaudiológico para identificar os princípios estruturantes que regem o funcionamento da técnica do atendimento em grupo e para tal, apóia-se na proposta de Zimerman.

Esta autora toma o primeiro fundamento técnico de Zimerman, a saber, o planejamento do atendimento onde o terapeuta deve realizar as seguintes perguntas: "Quem vai coordenar o grupo? Qual a finalidade do grupo? Como ele funcionará? e Quais circunstâncias e recursos?". O segundo fundamento seleção e agrupamento dos pacientes, integra o diagnóstico para que se indique o tipo de atendimento, relacionado com a finalidade, a constituição e o funcionamento deste grupo. Para a formação dos grupos, a autora leva em conta aspectos como coincidência de horários e faixa etária, porém pela imprevisibilidade inerente ao grupo, seria no decorrer de seu funcionamento que a fonoaudióloga visualizaria as emergências do grupo. Portanto, acredita que os critérios devam ser flexibilizados, organizados e constituídos na prática. Para melhor entendimento da proposta, trago as palavras da autora:

\footnotetext{
"no qual caso o terapeuta acredite na facilidade dessa tarefa proveniente da aproximação de uma mesma patologia, este pode ser um critério para a formação dos agrupamentos; no entanto se o terapeuta acredita que o enriquecimento do grupo deve ser dado de acordo com o maior numero de diferenças possíveis, este é outro critério a ser tomado. Dessa forma é importante, por ser uma clínica que lida com a subjetividade, os critérios sejam flexibilizados, impedindo a rigidez" (PASSOS, ${ }^{11}$, p. 60).
}

A terceira recomendação técnica de Zimerman diz respeito ao estabelecimento de um enquadre e a necessidade de preservação do mesmo, e a autora pauta-se nos elementos: "grupo homogêneo ou heterogêneo, fechado ou aberto, limitação do tempo (ou tempo ilimitado), número de participantes, número de reuniões, tempo, entre outros". (p.61).

Discorda-se da autora quando a mesma refere o critério de faixa etária, uma vez que deste independem os deslocamentos do grupo. No entanto, concorda-se com a mesma quando refere a imprevisibilidade e a heterogeneidade da constituição do grupo, pois seria no seu percurso tocado pela prática fonoaudiológica e pela sua configuração estrutural que se dariam os deslocamentos subjetivos e a coleta de subsídios para que o fonoaudiólogo se atente ao funcionamento deste grupo terapêutico.

A terceira e última categoria é denominada a função do fonoaudiólogo no atendimento em grupo. Esta, por sua vez, aponta para a configuração da posição do fonoaudiólogo no atendimento em grupo.

A maior parte dos trabalhos dedica-se a atribuir papéis ao fonoaudiólogo. Para Freitas, Lacerda e Panhoca ${ }^{2}$, o fonoaudiólogo teria o papel de interlocutor ativo; Panhoca ${ }^{3}$ e Panhoca e Leite ${ }^{4}$ Ihe atribuem o de coordenador e mediador; Hugenneyer e Oliveira ${ }^{9}$ preferem mediador; Panhoca e Penteado ${ }^{6}$, participante-coordenador; Silva et al ${ }^{13}$ o de eliminar e diminuir a patologia; Penteado et $a /^{5}$ consideram o fonoaudiólogo como agente, Mourão et $a l^{7}$ colocam-no no papel de propiciar alternativas para a produção de uma nova voz; Vilela e Ferreira ${ }^{14}$ afirmam que o fonoaudiólogo deve assumir o papel de auxiliador; Freitas e Dainês ${ }^{12} \mathrm{o}$ de mostrar as diferentes possibilidades de linguagem e Machado, Berberian e Massi ${ }^{8}$ declaram que o fonoaudiólogo deveria ser tocado pela escuta e com um auxílio de uma co-terapeuta.

Diante de outros pressupostos, Friedman e Passos $^{10}$ dizem que o terapeuta tem o papel de atuar ao mesmo tempo tanto no indivíduo quanto no conjunto formado por esses indivíduos. Passos ${ }^{11}$ traz o fonoaudiólogo para a posição de intérprete em relação aos acontecimentos e funcionamentos discursivos do grupo, porém com os atributos para ser um coordenador de grupo.

Finalizando a categoria função do fonoaudiólogo no atendimento em grupo, vários são os papéis do fonoaudiólogo (coordenador, participante- coordenador, mediador, agente, interlocutor ativo) no atendimento em grupo, porém, nestes papéis, os fonoaudiólogos focalizam os objetivos de cada sessão, com regras de convivência grupal e rotinas que levam os membros do grupo a se organizarem. Diante disso se poderia perguntar que espaço o 
fonoaudiólogo, ocupando esses diferentes papéis, dá aos sujeitos do grupo, se o foco do trabalho concentra-se nos objetivos de cada sessão, nas regras e rotinas? Qual a finalidade de utilizar essas regras para que os elementos do grupo sejam organizados? Neste caso, o fonoaudiólogo não se debruça sobre o funcionamento da linguagem do ser falante e nem o reconhece como aquele que faz parte de uma estrutura movida pela língua, pela escrita e pela fala. Nesses diferentes papéis, o fonoaudiólogo se coloca no lugar daquele que sabe, que atribui significados e pontua atitudes, como se essas intervenções pudessem incluir os sujeitos nos dizeres que circulam no grupo e proporcionar seu deslocamento pela circulação dos mesmos dizeres.

\section{CONCLUSÃO}

Conclui-se que o levantamento bibliográfico sobre o atendimento em grupo na área da
Fonoaudiologia identificou diferentes práticas fonoaudiológicas em grupo, atreladas a diversas perspectivas teóricas. Isto não quer dizer que haja consenso entre a atuação fonoaudiológica e a adoção desse tipo de prática em interface com a perspectiva que a embasa.

No que diz respeito aos critérios de formação, à função do fonoaudiólogo no grupo, à prevalência do atendimento em grupo em relação ao individual, houve divergências de opiniões, visto que a revisão da literatura fonoaudiológica, não (cor)respondeu com a consistência teórica esperada, porque há uma certa adoção irrefletida de conceitos de outras áreas, sem sustentação e sem a reestruturação exigida pela transposição do campo original pelo da clínica fonoaudiológica. Sugere-se que estas incoerências ocorrem porque não se evidencia a preocupação dos autores em articular os estudos teóricos aos elementos que configuram a clínica fonoaudiológica, a saber: a semiologia, a etiologia, a diagnóstica e a terapêutica.

\begin{abstract}
Background: the theme of this literature review is the group therapy in the Speech Therapy area. To perform it, we studied the texts published in scientific journals of the field, in the last 15 years, in the form of articles, book chapters and dissertations, which were focused on the group therapy. Purpose: the goal is to identify, understand and discuss, the following themes: 1) the theoretical principles that justify the indication of group therapy, structuring and maintaining it in order to explain how the dynamics of the group may act on the symptoms; 2) the criteria for the composition and formation of groups and especially, 3) the intervention of speech therapists and the possibility of their therapeutic effectiveness. Conclusion: it was concluded that the literature review on group therapy in the area of Speech Therapy identified different practices, linked to different theoretical perspectives. Regarding the criteria for group formation, the function of the speech therapist in the group, the prevalence of the group intervention in relation to the individual intervention, and there were differences of opinion since the review of the literature did not correspond to the expected theoretical consistency. This happened because there is a certain reflected adoption of concepts from other areas, without argumentation and without a restructuring demanded by the transposition to the speech therapy clinic. This is because there is no evident concern to articulate the theoretical studies to the elements that make up the speech therapy clinic, namely: the semiology, the etiology, the diagnosis and the therapeutics.
\end{abstract}

KEYWORDS: Speech-Language and Hearing Sciences; Language Disorders; Language Therapy; Peer Group

\section{REFERÊNCIAS}

1. Corrêa MB Considerações sobre a terapia de grupo na clínica fonoaudiológica. In: Lier-De Vitto MF, organizadora. Fonoaudiologia: no sentido da linguagem. São Paulo: Cortez; 1994. p.39-48.
2. Freitas AP, Lacerda MC, Panhoca I O grupo terapêutico fonoaudiológico - ensaios preliminares. Rev Bras Fonoaudiol; 1999; 7(1): 57-64.

3. Panhoca I O grupo terapêutico-fonoaudiológico e a literatura infantil - constituindo um saber. Rev Dist Comun, 1999, 11(1) : 29-57. 
4. Panhoca I, Leite APD A constituição de sujeitos no grupo terapêutico fonoaudiológico - identidade e subjetividade no universo da clínica fonoaudiológica. Rev Dist Comun: 2003, 15(2) : 289-308.

5. Penteado, RZ et al. Grupalidade e família na clínica fonoaudiológica: deixando emergir a subjetividade. Rev Dist Comun.2005; 17(2): 161-71. 6. Panhoca, I, Penteado RZ Grupo terapêutico-fonoaudiológico: a construção (conjunta) da linguagem e subjetividade. Pró-Fono; 2003; 15(3):259-66.

7. Mourão, LF et al. Grupo terapêutico-fonoaudiológico desenvolvido junto a laringectomizados totais: experiência em situação de Clínica Escola. Rev Dist Comun:2006, 18 (1): 51-61.

8. Machado M, Berberian A, Massi, G A terapêutica grupal na clínica fonoaudiológica voltada à linguagem escrita. In: Guarinello A, Santana A, Berberian A, Massi G (orgs.). Abordagens Grupais em Fonoaudiologia: contexto e aplicações. São Paulo: Plexus; 2007. p. 58-79.

9. Hugenneyer A, Oliveira S Terapia fonoaudiológica em grupo: um caminho possível. Rev Bras Fonoaudiol: 2000; 6:19-23.
10. Friedman S, Passos, $M O$ grupo terapêutico em fonoaudiologia: uma experiência com pessoas adultas. In: Guarinello A, Santana A, Berberian A, Massi G (orgs.) Abordagens Grupais em Fonoaudiologia: contexto e aplicações. São Paulo: Plexus; 2007. p. 138-63.

11. Passos, MC Atendimento fonoaudiológico em grupo: princípios estruturantes de uma técnica terapêutica [Dissertação de Mestrado ] São Paulo (SP): PUC, 2004.

12. Freitas AP, Dainês D Possibilidades de comunicação de um jovem com síndrome de Down durante o trabalho terapêutico-fonoaudiológico em grupo. Rev Bras Fonoaudiol: 2006: 1(3): 135-213.

13. Silva, FP et al. Organização de grupos terapêuticos no serviço público municipal de São José dos Campos. Fonoaudiologia Brasil: 2003: 2 (3): 22-7.

14. Vilela FCA, Ferreira LP. Voz na clínica fonoaudiológica: grupo terapêutico como possibilidade. Rev Dist Comun: 2006: 18(2): 235-43.

RECEBIDO EM: 13/08/2010

ACEITO EM: 01/10/2010

Endereço para correspondência:

Regina Maria Freire

Rua Heitor de Andrade, 170

São Paulo - SP

CEP: 05441-020

E-mail: freireregina@uol.com.br 